\title{
Propuesta de metodología para el monitoreo y seguimiento del Programa Productivo Alimentario en Nicaragua: Matriz de Marco Lógico
}

\author{
Tania López
}

Universidad de Chile

\section{Resumen}

El Programa Productivo Alimentario, mejor conocido como Hambre Cero, es un programa social implementado en Nicaragua desde 2007 por el Gobierno de Reconciliación y Unidad Nacional con el objetivo de reducir la extrema pobreza, el hambre y la desnutrición. La entrega de un bono no monetario a mujeres de áreas rurales de todo el país pretende fortalecer el rol de éstas en la economía comunitaria, dotándolas de capacidades para dar sustento a sus familias y organizándolas para comercializar sus excedentes de producción a fin de lograr un impacto económico y social a nivel nacional. El presente estudio tiene como objeto principal proponer el diseño de un sistema de monitoreo y seguimiento basado en la metodología de marco lógico, tomando como insumo el diseño original del programa y su actual funcionamiento. La contribución de este estudio responde a la inexistencia de una matriz que evalúe, continua y constantemente, el efectivo cumplimiento de los objetivos que permitan mejorar la gestión de recursos y de las instituciones involucradas.

Palabras clave: Monitoreo de políticas públicas, políticas nutricionales, superación de la pobreza.

\section{Proposal of methodology for the monitoring and follow-up of the Productive Food Program in Nicaragua: Logical Framework Matrix}

\begin{abstract}
The Food Production Program is a social program implemented in Nicaragua since 2007 by the Government of Reconciliation and National Unity in order to reduce extreme poverty, hunger and malnutrition. The delivery of a non-cash bonus to women in rural areas across the country intends to strengthen the role of them in the economy of the community, providing them with the capacity to support their families and organizing their surplus production to make an economic and social impact, in a national level. The main purpose of this study is to propose the design of a monitoring and tracking system based on the logical framework methodology, taking as input the original design of the program and its actual functioning. The contribution of this study responds to the absence of a matrix to assess, continually and constantly, the fulfilment of the objectives to improve the management of resources and institutions involved.
\end{abstract}

Keywords: Public policy monitoring, nutrition policy, overcoming poverty.

\footnotetext{
*Dirección de correspondencia [Correspondence

address]: Tania López, Universidad de Chile

E-mail: tanikari.lopez@gmail.com
} 


\section{Introducción}

Nicaragua cuenta con un ambiente macroeconómico positivo en las últimas décadas. Sin embargo, el bienestar de los pobres no tuvo cambio alguno en el período comprendido entre 1998 y 2005 (FIDEG, 2012). Para el año 2005, la incidencia de la pobreza general en Nicaragua se estimó en $48.3 \%$ y la pobreza extrema en $17.2 \%$. En contraste con años anteriores, ambos porcentajes no muestran variaciones significativas, por lo que se evidencia que Nicaragua no ha presentado cambios sustantivos en su meta de reducción de la pobreza.

De 1998 al 2005, la pobreza en los hogares del país pasó de $38.8 \%$ al $39.2 \%$. No obstante, entre el 2001 y 2005 se aprecia un aumento singular de 2.6 puntos porcentuales, ocurriendo lo mismo en los hogares pobres con niños (FIDEG, 2012). Según cifras oficiales de la FAO, Organización de Naciones Unidas para la Agricultura y la Alimentación, en Nicaragua en el período de 2003-2005 un $22 \%$ de la población total era sub-nutrida (Food and Agriculture Organization, 2012), esto es 1.2 millones de personas.

El acceso a la alimentación que le permita al individuo desarrollarse de acuerdo a sus capacidades es uno de los derechos fundamentales establecidos en la Carta de las Naciones Unidas sobre los Derechos Humanos y es el primer Objetivo de Desarrollo del Milenio, relacionado específicamente con la reducción de la extrema pobreza y erradicación del hambre.

Desde 2007, cuando el partido de Izquierda gana las elecciones con Daniel Ortega en la presidencia ${ }^{1}$, la principal preocupación del gobierno se centra en la reducción de la pobreza a través de programas sociales con focalización multisectorial directamente ejecutados y monitoreados por el Estado. A partir del programa de gobierno nace el Programa Productivo Alimentario del Ministerio Agropecuario y Forestal (MAGFOR), que se define como la "expresión concreta, clara y precisa" del actual gobierno de Nicaragua para combatir la pobreza en todas sus expresiones.

Este programa no tiene claridad sobre los diferentes objetivos a los cuales va contribuir y de los que se va hacer cargo. En el diseño del programa se plantean objetivos generales y específicos, pero no existe una herramienta de apoyo al monitoreo y seguimiento del mismo. Es por esto que esta inves-

\footnotetext{
${ }^{1}$ El programa de gobierno del FSLN prometía más inclusión social por medio de programas que beneficien a los más empobrecidos, apuntaba a reducir la pobreza por medio del desarrollo humano, dando un papel fundamental a las pymes y al rol de las mujeres en la sociedad.
}

tigación tiene como objetivo construir una Matriz de Marco Lógico para el Programa Hambre Cero, a fin de identificar los objetivos que debería cumplir en aras de contribuir al monitoreo y seguimiento del mismo.

\section{Instrumentos de monitoreo}

El enfoque top-down enfatiza la decisión creadora de la tecnocracia gubernamental y sus diseñadores con capacidad de generar programas y políticas que se implementan por derrame causal; la jerarquía, dirección y control se constituyen los elementos principales a la hora de comprender el proceso de instrumentación de políticas públicas. La jerarquización alude principalmente a la importancia y la influencia de la escala de poder que tienen los distintos niveles políticos y burocráticos sobre la implementación, es decir la mayor responsabilidad recae sobre la cúspide; la dirección y control también están ligadas a esta estructura jerárquica. Quienes planean y estructuran las políticas son responsables de su éxito, valiéndose de su capacidad directiva y de control sobre los niveles inferiores (Sabatier, 1986)

Este enfoque racionalista se expresa operativamente en la definición desde arriba de objetivos claros, estructura de órdenes hacia abajo debidamente prevista, aseguramiento de los recursos y apoyo necesario así como un sistema de comunicación que garantice el descenso de las órdenes centrales y la retroalimentación de los responsables.

Si se cumpliesen a cabalidad las características de esta visión de implementación de políticas públicas sería una estructura funcional casi perfecta. Pero el Estado no es "todo poderoso" y la racionalidad de las decisiones está condicionada por aspectos políticos fundamentales, dejando de lado la estructuración legal del proceso de implementación. Así, como propone Sabatier (1986), se trata de adoptar los elementos más fuertes del top-down y bottonup (enfoque botton-up, sobre enfatiza el poder de la periferia: al contrario del top-down). De este último su unidad de análisis, el estudio detallado de las estrategias de los actores intermedios y los más importantes en la escala de órdenes de la implementación; del primero su interés en conocer el impacto de las estructuras legales sobre la conducta de los actores, y la aplicación de esta perspectiva al estudio del cambio en las políticas en el largo plazo.

Como el sistema de aplicación de políticas públicas tiene fallas y la mayoría de estructuras obedecen más a enfoques verticalistas, lo importante es tener herramientas que ayuden a quienes ejecutan la política, y a la sociedad en general, a fortalecer 
el papel que estos juegan en el ámbito de políticas públicas.

Una herramienta fundamental, son los instrumentos de monitoreo. Estos dan lugar a una adecuada toma de decisiones a partir del análisis de resultados obtenidos, permiten medir el grado de eficiencia y efectividad de los programas para cumplir los objetivos para los cuales fueron creados. No se puede hablar de monitoreo dejando de lado la evaluación, ya que son procesos que van de la mano y que constituyen una fuente primordial de aprendizaje para los gobiernos y gestores de políticas públicas (Subirats, 1995). Sin embargo, el monitoreo, a diferencia de la evaluación, es el seguimiento que se lleva a cabo durante la ejecución de una política, programa o proyecto. Es un proceso permanente y continuo, un instrumento de gestión que revisa en forma periódica los aspectos fundamentales de las tres P (Políticas, Programas y Proyectos).

El objetivo principal de monitorear es "indagar y analizar permanentemente el grado en que las actividades realizadas y los resultados obtenidos cumplen con lo planificado, con el fin de detectar a tiempo eventuales diferencias, obstáculos o necesidades de ajuste en la planificación y ejecución" (UNESCO y SIEMPRO, 1999)

Un sistema de monitoreo también es una herramienta importante para conseguir financiamiento con donantes. Este aspecto es particularmente importante para la realidad nicaragüense, teniendo en cuenta que el 2,2\% del PIB en el 2013 (Banco Central de Nicaragua, 2013) proviene de donaciones, y que los donantes requieren conocer en qué se gastan los recursos, la transparencia con que lo hacen y sobre todo si finalmente causan impacto en la población.

\section{Matriz de Marco Lógico}

Es una herramienta para facilitar el proceso de conceptualización, diseño, ejecución y evaluación de proyectos. Su propósito es brindar estructura al proceso de planificación y comunicar información esencial relativa al proyecto.

La Matriz de Marco Lógico que se elabora para efectos de la evaluación debe reflejar lo que el programa es en la actualidad. Si bien muchos programas no han sido diseñados con el método del Marco Lógico, se debe realizar un ejercicio de reconstrucción de los distintos niveles de objetivos del programa (fin, propósito, componentes) con sus respectivos indicadores, que permitan medir el nivel de logro alcanzado. La Institución Responsable del programa debe completar cada casilla de la matriz.
- Fin del Programa: Es la descripción de cómo el programa contribuye, en el largo plazo, a la solución del problema o satisfacción de una necesidad que se ha diagnosticado. No implica que el programa, en sí mismo, será suficiente para lograr el Fin, pudiendo existir otros programas que también contribuyen a su logro.

- Propósito del Programa: Es el resultado directo a ser logrado en la población objetivo, como consecuencia de la utilización de los componentes (bienes y/o servicios) producidos por el programa. Es una hipótesis sobre el beneficio que se desea lograr. Es la contribución específica a la solución del problema (o problemas) diagnosticado (s).

- Componentes del Programa: Son los bienes y/o servicios que produce o entrega el programa para cumplir su propósito. Deben expresarse como un producto logrado o un trabajo terminado (sistemas instalados, población capacitada, alumnos egresados, etc). El conjunto de los componentes permite el logro del propósito. Un componente es un bien y/o servicio dirigido al beneficiario final o, en algunos casos, dirigido a beneficiarios intermedios. No es una etapa en el proceso de producción del componente.

- Actividades del Programa: Son las principales tareas que se deben cumplir para el logro de cada uno de los componentes del programa. Corresponden a un listado de actividades en orden cronológico y secuencial, para cada uno de los componentes. Las actividades deben presentarse agrupadas por componente.

- Indicadores: Los indicadores de desempeño son una herramienta que entrega información cuantitativa respecto del nivel de logro alcanzado por un programa, pudiendo cubrir aspectos cuantitativos o cualitativos de este logro. Es una expresión que establece una relación entre dos o más variables, la que comparada con períodos anteriores, productos (bienes o servicios) similares o una meta o compromiso.

La Matriz de Marco Lógico de un Programa presenta un diseño coherente cuando se cumple su lógica horizontal y vertical.

- Lógica Horizontal de la Matriz de Marco Lógico: Se basa en el principio de la correspondencia, que vincula cada nivel de objetivo (fin, propósito, componente y/o actividades) a la medición del logro (indicadores y medios de verificación) y a los factores externos que pueden afectar su ejecución y posterior desempeño (supuestos principales). 
- Lógica Vertical de la Matriz de Marco Lógico: se basa en relaciones de causa-efecto entre los distintos niveles de objetivo de la Matriz de Marco Lógico y postula lo siguiente:

Si se realizan las actividades se producen los componentes, siempre y cuando los supuestos identificados sean confirmados en la realidad de la ejecución del programa. Las actividades necesarias y suficientes para producir un componente, junto con los supuestos a este nivel, confluyen al éxito de la ejecución del mismo.

Si se producen estos componentes y los supuestos de este nivel se ratifican, se logrará el propósito. Los componentes, junto con los supuestos a este nivel, describen las condiciones necesarias y suficientes para lograr el propósito. Si se logra el propósito, y se confirman los supuestos de este nivel, se habrá contribuido de manera significativa a alcanzar el fin. El propósito, junto con los supuestos a este nivel, describen las condiciones necesarias, aun cuando no sean suficientes, para lograr el fin.

\section{Monitoreo en Nicaragua}

En Nicaragua la falta de recursos económicos limita en muchas ocasiones el asignar presupuesto para fortalecer los sistemas de monitoreo de programas y proyectos sociales principalmente, y es difícil saber qué obstáculo representa una mayor amenaza para de desarrollo de estos procesos.

La realidad nacional evidencia un claro problema de acceso a la información así como de disponibilidad de la misma. Esto tiene que ver la poca inversión en sistemas de información, líneas de base, y con la transparencia de las instituciones para publicar información, sobre todo cuando hay compromisos de cumplir indicadores para obtener donaciones y financiamientos.

Por otro lado, son pocas las instituciones que ponen a disposición del público sus archivos y bases de datos, no necesariamente por falta de voluntad pero sí por falta de personal capacitado que procese la información y tecnología de información que permita actualizar las plataformas virtuales de las instituciones. El Banco Central de Nicaragua y Ministerio de Hacienda son de las pocas instituciones que sí cuentan con un excelente sistema actualizado y disponible en la web. Este es un esfuerzo respaldado por la cooperación internacional que busca fomentar la cultura de monitorear los indicadores más importantes de crecimiento y desarrollo económico, así como el desglose del presupuesto general de la república.
Sin embargo, la mayoría de programas sociales no tienen un sistema de información actualizado y en algunas ocasiones no lo tienen del todo. En el caso del PPA, la información no está sistematizada, no hay un consenso dentro de las instituciones que implementan el programa en cuanto a muchos de los datos estadísticos; y principalmente no tiene un instrumento que permita dar continuo seguimiento a los objetivos que intenta cumplir el programa para mejorarlo.

En Nicaragua se trabaja muy poco con enfoque de marco lógico. Es más común su uso en las ONGs nacionales y sobre todo en las de cooperación internacional; se conoce la técnica, pero no se ha llevado a la práctica en las instituciones públicas y por esta razón no se conocen sus facilidades. Es el BID el que generalmente en sus formas de trabajo conjunto solicita el uso de marco lógico.

La herramienta más utilizada para monitorear las actividades de los programas en las organizaciones es el Plan Operativo Anual, que si bien es un sistema eficiente en el corto plazo focalizando la asignación de recursos humanos y materiales, no facilita la creación de indicadores que permitan evaluar el cumplimiento de objetivos de manera constante.

Un programa que tuvo un sólido sistema de evaluación y monitoreo es la Red de Protección Social, a la que sustituyó al PPA, realizado por el Banco mundial, CEPAL y organizaciones que promueven políticas de salud y nutrición ${ }^{2}$.

\section{Descripción del Programa Productivo Alimentario}

El Programa Productivo consiste en la entrega de un bono productivo, es decir bienes (cerdas y vacas preñadas, gallinas, semillas, etc.), acompañado de asistencia técnica para asegurar la sostenibilidad de la producción y cambios de comportamiento dentro de las familias rurales a nivel nacional. Estamos hablando "de una economía diversificada de reproducción ampliada que genera y reinvierte excedentes, que comercializa y escala mercados, que incorpora tecnología para incrementar rendimientos, que tiende a industrializarse ya mejorar su nivel de vida año con año, contribuyendo así al mejoramiento de la economía nacional." (MAGFOR, 2008: 10)

En el diseño, se trata de una transferencia en forma de bienes y animales que posteriormente deben reintegrar hasta en un $20 \%$ aproximadamente, para lograr la sostenibilidad del programa de manera que se pueda beneficiar a otras familias por vía am-

${ }^{2}$ IFPRI: International Food Policy Research Institute 
pliación de cobertura.

El objetivo y meta del programa es "Erradicar el hambre, la desnutrición crónica, la pobreza extrema y el desempleo en 75,000 familias rurales pobres, mediante el incremento cuantitativo y cualitativo de la producción y el consumo de alimentos proteicos, favoreciendo a la vez la sustitución del consumo de leña por el biogás" (MAGFOR, 2008: 15)

Desde el punto de vista organizativo, el programa tiene cuatro momentos: En un primer momento se seleccionan y se organizan las familias para recibir el bono de capitalización. En un segundo momento las familias en número de 50 forman una cooperativa. En un tercer momento, varias cooperativas conforman una unión o central de cooperativas. En un cuarto momento las uniones o centrales conforman federaciones de cooperativas.

En cuanto a la parte productiva también se divide en 4 momentos; la primera fase o momento corresponde a la ejecución del Bono Productivo Alimentario y está a cargo del Programa Productivo Alimentario del MAGFOR (hoy en manos del ministerio de Economía Familiar, Comunitaria, Cooperativa y Asociativa). La segunda fase o momento será apoyada en lo que a manejo de fondos se refiere por el Fondo de Crédito Rural (FCR). La tercera fase o momento será apoyada en lo que a agroindustria se refiere por el Instituto de Desarrollo Rural (IDR). La cuarta fase será apoyada por el Ministerio de Economía, el Banco de Fomento, la Cancillería de la República, la Secretaría Técnica de la Presidencia, entre otras.

Ahora bien, dado que actualmente existen otras familias campesinas (distintas a las familias beneficiarias del bono productivo) que ya se encuentran en la segunda, tercera y cuarta fase; las mismas estarán siendo atendidas desde ahora por las instituciones señaladas. Asimismo, participan en la atención a las familias campesinas en general otras instituciones agropecuarias, tales como el Instituto de Tecnología Agropecuario (INTA), el Instituto Nacional Tecnológico (INATEC), la Empresa Nacional de Abastecimiento (ENABAS), además de los organismos encargados de atender y fomentar el cooperativismo en el país. En su cuarta fase las uniones o federaciones cooperativas deberán ser atendidas por el Banco de Fomento y demás servicios bancarios públicos y privados, por el ministerio de Finanzas, Industria y Comercio (MIFIC) por todas las otras instituciones relacionadas con el desarrollo nacional, particularmente por la iniciativa Alternativa Bolivariana de las Américas (ALBA).

El programa se centra en productores empobrecidos porque "los pequeños y medianos productores del campo son los mayores generadores de empleo, riqueza y producción exportable, de donde resulta que invertir en ellos es económicamente rentable" 3

Este programa se ha focalizado exclusivamente en mujeres pobres campesinas, ya que desde la percepción de éste y basados en la observación empírica, son quienes tienen más sentido de responsabilidad a la hora de administrar el presupuesto familiar.

La atención de 75,000 mujeres a quienes se les entrega el bono y quienes se organizan para administrarlo, se convierte en el mayor programa de cooperativismo de mujeres y por lo tanto en el mayor programa de liderazgo de la mujer en el campo nicaragüense.

Una vez bien instalado el Gobierno de Unidad y Reconciliación Nacional de inmediato se inició el proceso de implementación del programa, sin un presupuesto definido tampoco quedaba claro el papel que jugaría respeto de la política rural en general y la política de seguridad y soberanía alimentaria y nutritiva.

\section{Metodología}

En función de contar con un diagnóstico del programa para poder diseñar una Matriz de Marco Lógico para el PPA, se elaboró un cuestionario basado en la estructura de la matriz con el objetivo de obtener información precisa sobre los componentes y actividades. Dicho cuestionario fue enviado a tres funcionarios públicos que trabajaron de cerca con el programa y a dos investigadoras de la sociedad civil que realizaron un estudio sobre la implementación y transparencia del PPA. La selección de estos actores responde a la revisión del mapeo de actores, a la conveniencia y a la accesibilidad, dado que éstos se encuentran en Nicaragua y este estudio se ha realizado en Chile.

Dado que se habla de un programa que ya se encuentra en marcha desde el 2007, no se evalúa la decisión de llevarlo a cabo, sino que se diseña un método para dar seguimiento mediante indicadores medibles y verificables usando el diseño del programa existente para identificar los principales objetivos. Es lo que se conoce como diseño inverso de la matriz de marco lógico (Aldunate, 2011).

Además, siguiendo la metodología de marco lógico, se elaboraron los árboles de problema y de objetivos a partir del problema que el programa intenta

\footnotetext{
${ }^{3}$ Palabras de Orlando Núñez en entrevista exploratoria para este estudio de caso. Enero 2014
} 
resolver, haciendo revisión de información de la pobreza en Nicaragua. La elaboración de alternativas a partir de los árboles no fue incorporada ya que no se está evaluando la formulación del programa, sino durante la implementación qué objetivos se desean alcanzar y cómo se podrían medir.

\section{Diagnóstico y propuesta de Ma- triz de Marco Lógico}

\section{Actores involucrados ${ }^{4}$}

\section{Gobierno}

La estrategia de ejecución del Bono es responsabilidad del Gabinete Nacional de Producción, conducido por la Presidencia de la República. La institución del Gabinete de Producción que coordina este programa es el MAGFOR (Ministerio Agropecuario y Forestal), entregando las tecnologías y orientando sus acciones al desarrollo de capacidades técnicas y productivas para mejorar la seguridad alimentaria de las familias beneficiadas.

Las instituciones del Gabinete Nacional de Producción que participan para impulsar, complementariamente el desarrollo asociativo de las beneficiarias en sus comunidades y sus capacidades en el procesamiento y la comercialización de los excedentes son:

- Instituto Nicaragüense de Tecnología Agropecuaria (INTA), en el marco del fortalecimiento del modelo de desarrollo agropecuario esta institución se propone reorientar la estrategia de trabajo, desarrollando la investigación e innovación a fin de incrementar la producción y productividad principalmente de pequeños y medianos productores/as del país.

- GRUN (Gobierno de Reconciliación y Unidad Nacional), a través de la Primera Dama: Secretaria de Comunicación. Fomenta y establece como uno de los ejes principales de su política de soberanía alimentaria. Baja los lineamientos hacia las instituciones ejecutoras.

- El Instituto de Desarrollo Rural (IDR), es una Institución pública del Poder Ciudadano que facilita servicios de apoyo a la producción, con el fin de garantizar la seguridad alimentaria de la población rural pobre.

\footnotetext{
${ }^{4}$ La información general de las "instituciones participantes del PPA" se proporcionó en archivos que no se encuentran en la web, complementada con informe de Avances-Desafíos de Hambre Cero de IEEPP.
}

- El Instituto Nicaragüense de Fomento Cooperativo (INFOCOOP), es el organismo rector de la política nacional de protección, fomento y desarrollo cooperativo, además de la regulación, suspensión, supervisión y control de las cooperativas. Tiene como objetivo principal fomentar, promover, divulgar y apoyar el movimiento cooperativo a todos los niveles.

- El Fondo de Crédito Rural (FCR) del Banco Produzcamos y el Instituto Nacional Tecnológico (INATEC), brindan acompañamiento financiero y capacitación técnica para mejorar los sistemas productivos que dinamicen la producción.

- INIFOM (Instituto Nicaragüense de Fomento de Municipios), se encarga de comprar y entregar bienes a las familias que habían sido seleccionadas según los criterios correspondientes.

- Parlamento Nacional, aprobó presupuesto para el MAGFOR y el Ministerio de Economía Familiar así como dentro de la ERCERP (Estrategia de Reducción de la Pobreza) para la implementación del PPA. Cabe mencionar que al pasar de 2007 al 2008 el presupuesto se vio incrementado de C\$183 millones a C\$ 264 millones de córdobas, los siguientes años mantuvo un presupuesto alrededor de los C\$200 millones.

- CGR (Contraloría General de la República), forma parte del aparato estatal, es auditor de procedimientos legales/presupuestario en cuanto a licitaciones para llevar a cabo el programa. La CGR excluyó de trámites de licitación al PPA en los primeros meses de su implementación.

- También se integran en la ejecución -para asegurar los objetivos del programa- las Alcaldías y estructuras del Poder Ciudadano en los territorios, ellos en el terreno son los más cercanos a la comunidad e inciden en la selección de beneficiarias.

\section{Sociedad Civil}

- CIPRES (Centro para la Promoción, la Investigación y el Desarrollo Rural y Social) Es la Organización que implementó el proyecto Piloto de la cual se desprendió Hambre Cero, las experiencias exitosas fueron utilizadas en el Programa nacional. Actualmente no se encuentra vinculada

- CPC (Consejos del Poder Ciudadano) Asociación de vecinos pertenecientes al partido de gobierno quienes tienen mucha presencia política 
en los barrios y han influido en la selección de usuarias del programa así como los GPC (Gabinetes del Poder Ciudadano) que también coordinan con las instituciones para la distribución del Bono.

Las beneficiarias directas así como sus familias son el objetivo principal del programa y claramente son participantes activos que van configurando el PPA. Para la selección de las beneficiadas se ha montado una estructura a nivel departamental, municipal y comarcal, donde participan representantes del MAGFOR, los Consejos del Poder Ciudadano (CPC) y los secretarios políticos del FSLN. Tomando en cuenta el número reducido de Bonos y el gran número de familias rurales y semi-urbanas que viven en extrema pobreza, la selección de las beneficiadas se convierte en un componente sensible

\section{Organismos Internacionales}

- FAO (Food and Agriculture Organization) por medio de la Organización de Naciones Unidas y los Objetivos de Desarrollo del Milenio tiene especial interés en la seguridad alimentaria y nutrición de los países. Monitorea las acciones del gobierno en cuanto a estos temas, las instituciones brindan la información a discreción. FAO contribuyó significativamente en la financiación y diseño del programa y el Fondo de las Naciones Unidas para la Infancia (UNI$\mathrm{CEF}$ ) y es un actor indirecto en la medida en que vela por los derechos de la niñez, en este caso la seguridad nutricional, mediante el código de la niñez y las normativas en la legislación nicaragüense.

- BID (Banco Interamericano de desarrollo), la cooperación Taiwanesa y austriaca hicieron significativos préstamos y donaciones según cada caso para lograr los objetivos del programa.

Los fondos de financiamiento provienen, mayoritariamente, de los recursos del Presupuesto General de la República, complementándose con recursos externos de fuentes tales como Venezuela, BID, Taiwán, AECI/Fondos para el Desarrollo de los Objetivos del Milenio, Fondo Común (Suiza, Finlandia y Suecia) y Austria, así como fondos provenientes de programas dirigidos al combate a la pobreza, que administran y ejecutan instituciones públicas y asociadas del sector agropecuario, alineándose a la política y estrategia del Gobierno. De las rentas del tesoro se ha financiado el $40 \%$, de donaciones externas el $3 \%$ y de préstamos externos $57 \%^{5}$

\footnotetext{
${ }^{5}$ Información obtenida en página del Ministerio de Hacienda, MHCP, Presupuestos Generales de la República.
}

Los fondos de ALBA (Alternativa Bolivariana para Nuestros Pueblos de las Américas), ALBANISA (Alternativa Bolivariana para nuestros Pueblos de las Américas de Nicaragua Sociedad Anónima) así como de CARUNA (Caja Rural Nacional) esta última compuesta por fondos provenientes del ALBA, desembolsaron para los Bonos de Economía de Patio.

En el mapa de actores se distinguen a los interesados de los implicados en la Política Pública, y dentro de esas esferas se encuentra el foco central del programa, la población objetivo que requiere superar la pobreza y desnutrición.

El GRUN es quien implantó el programa presentado por Orlando Núñez de CIPRES, -el cual ya no tiene mucho que ver en el mismo-, dentro del set de políticas públicas de este gobierno. Este baja los lineamientos a los Ministerios ejecutores del PPA. El Ministerio de Hacienda según lo aprobado por el Parlamento en el presupuesto, asignaba al MAGFOR los montos correspondientes para ejecutar el programa, el cual compraba los bienes a entregar y se coordinaba con los demás ministerios implicados; actualmente es al Ministerio de Economía Familiar, Comunitaria y Asociativa a quien se traspasó la dirección del PPA. El MINSA y MINED tienen un rol menos activo que los demás ministerios, es posible que la etapa predominante actualmente sea la de distribución y asistencia técnica, pero en un largo plazo con la conformación de comunidades participativas y organización de cooperativas, se espera que se logre integrar el componente de salud y educación, tal como se tiene contemplado en el diseño del programa, sin embargo es un objetivo igualmente ambicioso que requiere más tiempo y cambio de comportamiento por parte de las familias.

Los organismos como FAO-UNICEF son observadores-evaluadores del programa, no participan directamente para el desarrollo de este, más si en alguna etapa del diseño dando ciertas recomendaciones al mismo para mejorar ciertos aspectos estructurales, en general las organizaciones de la ONU deben respetar la autonomía en las decisiones a lo interno de los países.

Se observa también que dentro de los involucrados, en alguna medida, los grupos están haciendo lo suyo por separado, existen diferentes niveles de incidencia. Por ejemplo, la cooperación venezolana tiene diferente acercamiento (fuerte relación) al gobierno del que tiene el Banco Mundial o el Banco Interamericano de Desarrollo, esto por los interesesafinidades políticas.

Por su parte, las organizaciones de la sociedad civil en su mayoría critican la implementación del 
programa en cuanto a la selección de las beneficiarias, la cobertura, así como la rendición de cuentas y ejecución del programa. También los medios de comunicación se encuentran parcializados, se puede distinguir entre los llamados "oficialistas" y los de oposición, en su mayoría no son medios fidedignos. Poco se puede hablar de periodismo investigativo (IEEPP, 2011).

Además, los CPC y GPC son estructuras organizacionales gestionadas por el FSLN por ende todas sus actividades son avaladas por el gobierno ${ }^{6}$. Están directamente relacionados con los usuarios así como las alcaldías que en su mayoría son de este actual gobierno. Estas asociaciones participan muy de cerca en la implementación del PPA.

No hay evidencia sobre hasta qué punto las mujeres beneficiarias y sus familias participaron en el diseño o construcción colectiva del programa, asimismo respecto al seguimiento que se les ha dado a estas familias y sus comunidades. Los usuarios del bono claramente, una vez que son electos, tienen una participación activa en la sostenibilidad que se pretende con el programa. Finalmente son ellos los testigos del impacto del programa en la calidad de vida de sus familias y el entorno en general.

\section{Matriz Diagnóstico}

Después de unos 7 años de implementación aproximadamente, y con aporte de apreciaciones de algunos actores relacionados al diseño y ejecución del PPA se puede extraer una especie de fotografía del estado del programa en cuanto a lo que ha sido:

Como consenso, dentro del documento que define el Programa Productivo Alimentario y en lo obtenido como respuesta en los cuestionarios, el fin del programa queda planteado como: "concebido con el objetivo de erradicar la pobreza extrema y el hambre en Nicaragua, priorizando a las mujeres rurales. El objetivo se lograría a través del otorgamiento de un conjunto de bienes [?] llamado Bono Productivo" (Cuestionario CG). Asimismo, el propósito tangible del PPA es "la capitalización de familias pobres en el campo y periurbana para elevar el nivel de vida mediante el incremento cuantitativo y cualitativo de la producción y el consumo de alimentos proteicos" (Cuestionario CM). Son 75 mil familias que fueron mencionadas cuando se describieron los objetivos de Hambre Cero, porque así lo especificó

\footnotetext{
${ }^{6}$ Los CPC y CPC son organizaciones legalmente reconocidas por el gobierno de Nicaragua, creado por la actual administración de Daniel Ortega, vistas como una extensión del partido político del presidente y están presentes a nivel nacional http://www.confidencial.com.ni/archivo/2009651/politica2_651.html
}

el MAGFOR en su momento como meta inicial.

Según el diseño del programa, se plantean diversos objetivos que en su mayoría no se logran cumplir:

"La mejoría de los ingresos familiares no se logra
porque lo que dieron no permitía vender produc-
tos, puesto que apenas daba para el consumo fa-
miliar. También se plantearon mejorar la equidad
de género en las familias y la distribución de ta-
reas entre hombres y mujeres; en este aspecto, no
sólo no se mejora, sino que se recarga a las muje-
res de más tareas y responsabilidades, incluyendo
la de disminuir la pobreza de la familia, la deuda
por las cosas que le entregaron, la obligación de
participar en reuniones, capacitaciones y en acti-
vidades políticas y organizativas; así como el cuido
y la responsabilidad de los animales entregados"

(Cuestionario AQ).

Esta aseveración es fruto de una investigación realizada por el Instituto de Estudios Estratégicos y Políticas Públicas (IEEPP) en terreno, que evidencia las deficiencias del programa desde las voces de algunas beneficiarias, entonces ¿qué tan conveniente es tener una metodología de monitoreo de objetivos para un programa que nace como un compromiso político?. Si la idea del programa es integrar a todos los actores sociales, es necesario que cuenten con una herramienta de fiscalización en cuanto a medir el cumplimiento de los objetivos.

Por otro lado, en ésta matriz diagnóstico no se evidencian supuestos ya que según un ex funcionario del Ministerio Agropecuario y Forestal:

\begin{abstract}
"No hay ninguna consideración en riesgo externos para su implementación. Esta fue una oferta de la campaña electoral que llevó al poder al FSLN en el 2007. O sea había un compromiso político. La fundamentación para su implementación descansaba en los éxito obtenidos por el CIPRES el en modelo implementado en sus programa a poblaciones rurales, principalmente después del Huracán Mitch de 1998, entre ellos en Chacraseca León"
\end{abstract}

(Cuestionario CM).

Sin embargo, efectivamente existen riesgos externos que no están en las manos del programa y que al ser independientes, este no se hace cargo. Es importante tenerlos en cuenta porque influyen en el desarrollo de Hambre Cero así como en el impacto que tiene en quienes se benefician de él.

Con relación a los indicadores, la existencia de información es casi nula o muy limitada. Lo que exis- 
Tabla 1: Matriz diagnóstico

\begin{tabular}{|c|c|c|}
\hline Objetivos & Indicadores & Verificadores \\
\hline $\begin{array}{l}\text { Fin: Contribuir a reducir la pobreza } \\
\text { extrema, el hambre y la desnutrición en } \\
\text { zonas rurales y peri-urbanas de Nicaragua }\end{array}$ & Mapa de pobreza. & $\begin{array}{l}\text { Sitio web INIDE, Mapa de pobreza extrema } \\
\text { según NBI. }\end{array}$ \\
\hline $\begin{array}{l}\text { Propósito: } 75 \text { mil familias rurales } \\
\text { capitalizadas, productivas y asociadas. }\end{array}$ & $\begin{array}{l}\text { Número de familias } \\
\text { beneficiadas con el BPA }\end{array}$ & $\begin{array}{l}\text { SPPA MAGFOR } \\
\text { VI objetivos del subprograma }\end{array}$ \\
\hline $\begin{array}{l}\text { Componente 1: Familias Rurales logran } \\
\text { producir más alimentos de mejor calidad y } \\
\text { asi asegurar su alimentación y nutrición. }\end{array}$ & $\begin{array}{l}\text { Productividad de granos } \\
\text { básicos. }\end{array}$ & $\begin{array}{l}\text { Página oficial Banco Central de Nicaragua: } \\
\text { Estadiaticas, anuario; Sector Real, I21 }\end{array}$ \\
\hline $\begin{array}{l}\text { Componente 2: Las mujeres jefas de hogar } \\
\text { forman asociaciones y comercializan sus } \\
\text { excedentes de producción. }\end{array}$ & $\begin{array}{l}\text { Número de núcleos de } \\
\text { mujeres asociadas }\end{array}$ & $\begin{array}{l}\text { Página oficial MEFCCA } \\
\text { PNDH Nicaragua: Hambre Cero }\end{array}$ \\
\hline $\begin{array}{l}\text { Componente 3: Niños con mejor nivel } \\
\text { educativo }\end{array}$ & Tasa de escolaridad. & $\begin{array}{l}\text { Ministerio de Educación, no disponible en } \\
\text { página web. Se solicita información de } \\
\text { manera personal. Información no } \\
\text { actualizada a la fecha en páginas de la } \\
\text { CEPAL }\end{array}$ \\
\hline $\begin{array}{l}\text { Componente 4: Niños con mejor nivel } \\
\text { nutricional. }\end{array}$ & $\begin{array}{l}\text { Nivel de nutrición en } \\
\text { menores de } 5 \text { años }\end{array}$ & $\begin{array}{l}\text { Estadisticas Ministerio de Salud } \\
\text { disponibles via solicitud escrita. } \\
\text { Censa Nacional de Nutrición. }\end{array}$ \\
\hline $\begin{array}{l}\text { Actividades Cl: Selección de usuarias y } \\
\text { entrega de Bono Productivo (Vacas, cerdas, } \\
\text { gallinas, semillas). }\end{array}$ & $\begin{array}{l}\text { Número de bonos } \\
\text { entregados a beneficiarias }\end{array}$ & Página oficial MEFCCA \\
\hline $\begin{array}{l}\text { Actividades C2: Capacitación técnica: } \\
\text { 1.aprovechamiento de los bienes } \\
\text { entregados 2.organización de asociaciones } \\
\text { de mujeres productoras y 3.capacitació } \\
\text { financiera para acceso a créditos } \\
\text { Creación de planes de negocios para la } \\
\text { comercialización de la producción. }\end{array}$ & $\begin{array}{l}\text { Número de mujeres } \\
\text { productoras capacitadas. } \\
\text { Cantidad de mujeres con } \\
\text { acceso a créditos bancarios. } \\
\text { Numero de planes de } \\
\text { negocio presentados. }\end{array}$ & $\begin{array}{l}\text { Seguimiento programa hambre cero. Página } \\
\text { oficial MEFCCA: Hasta } 2012 \\
\text { PNDH Nicaragua: Hambre Cero }\end{array}$ \\
\hline $\begin{array}{l}\text { Actividades C3: Las familias ge } \\
\text { comprometen a enviar a sus niños a la } \\
\text { escuela }\end{array}$ & $\begin{array}{l}\text { No existe un indicador que } \\
\text { este siendo monitoreado en } \\
\text { este sentido }\end{array}$ & \\
\hline $\begin{array}{l}\text { Actividades C4: Los niños de las familias } \\
\text { rurales asisten controles periódicos en } \\
\text { centros de salud. }\end{array}$ & $\begin{array}{l}\text { Tasa de variación de } \\
\text { desnutrición infantil. }\end{array}$ & \\
\hline
\end{tabular}

Fuente: GRUN (2012); Banco Central de Nicaragua (2005); Ministerio de Economía Familiar Cooperativa y Asociativa (2012)

te como indicadores son más bien datos estadísticos descriptivos:

"Lo cierto es que el gobierno no manifestó interés en evaluar o dar seguimiento con seriedad al programa y esto está demostrado por la falta de información o la multiplicidad de datos para un determinado período provenientes incluso de la misma fuente. (Se encontraron hasta 6 datos sobre beneficiarios con diferencias de hasta 50,000 personas)"

(Cuestionario AQ).

La inestabilidad institucional del programa, debido a conflictos internos, también limita la obtención de información clara y verificable, Hambre Cero ha estado en mano de distintas instituciones en el tiempo que lleva su implementación. Primero estuvo a cargo del Ministerio de Agrícola y Forestal y actualmente por el recién creado Ministerio de Economía Familiar, también hubo un desfile de directores del programa en los primeros años de éste, condición que debilitó en su momento la credibilidad del mismo, y que teniendo divididas las tareas, la sistematización de la información se vuelve engorrosa.

Ahora bien, para construir una Matriz alternativa que proponga un mejor camino hacia el monitoreo y seguimiento del programa, es necesario definir el árbol de problemas y su respectivo árbol de objetivos para el objetivo principal que como programa se propone Hambre Cero.

$\mathrm{Al}$ analizar el árbol de problemas y de objetivos, elaborados con algunos aportes de los cuestionarios, se puede obtener un sin número de objetivos que le competen al programa y otro que, si bien se desprenden del principal problema, el programa no puede hacerse cargo y se convierten en su gran 
mayoría en supuestos de la Matriz a proponer, por ejemplo la vulnerabilidad ante desastres naturales, los precios internacionales de granos básicos, entre otros.

En el árbol de problemas, los efectos corresponden a las consecuencias de la mala alimentación y el alto nivel de desempleo que por su falta de calificación tienen, unido a esto la baja comercialización de sus productos por falta de créditos, de capacitación en planes de negocio, cooperativas productivas, etc. Las causas tienen causas en sí mismas y son una cadena de condiciones.

En la propuesta de matriz sí se consideraron supuestos acordes al fin, propósito, componentes y actividades, aunque el PPA ya está siendo implementado, pues en teoría estos deberían cumplirse para que sea factible y se cumplan los objetivos del proyecto o programa, y por otro lado porque pueden estar explicando algunas falencias del éste.

El fin de Hambre Cero en su discurso es, como ya se ha mencionado, la reducción de la pobreza extrema, sin embargo, se le adiciona en "población rural" porque es ahí donde actúa directamente el PPA. Es en el área rural donde se agudiza la pobreza en el país, y las causas son innumerables, desde la falta de educación que perpetúa la situación y condición de vida de estas familias, hasta la poca inversión pública que limita las oportunidades y hace que los campesinos migren a las ciudades y abandonen su trabajo en la producción agrícola.

\section{Propuesta de Diseño de MML}

$\mathrm{Al}$ estudiar los árboles de problema y objetivos que se obtuvieron de la revisión del documento diseño original del programa, literatura de causas de la pobreza e información de los cuestionarios, se logra descomponer en objetivos - de forma generallo que Hambre Cero realmente va producir en las familias usuarias del bono que éste entrega.

El problema principal es la reducción de la pobreza, y se asume que la producción de alimentos que la familia va a obtener, se traduce en una mejor nutrición para los miembros de ésta, principalmente los niños.

Si bien la pobreza es un problema multidimensional, y las causas son numerosas, claramente el tema de la delincuencia y todo el ambiente que surge de la falta de oportunidades requiere de otras políticas que combatan directamente estas situaciones.

El papel de la educación es algo de lo cual no se puede hacer cargo el programa, pues tiene que ver con que realmente las usuarias cumplan el compro- miso de enviar a sus hijos a la escuela.

Además, los factores externos, como los climatológicos, que se encuentran fuera de las manos del programa, se consideraron supuestos porque finalmente sí influyen en la continuación del PPA y se deben tomar en cuenta para valorar las alternativas de acción.

De esta manera, la matriz de propuesta se centra en las usuarias del programa y lo que éste va producir para lograr cumplir el propósito y contribuir al fin.

El principal obstáculo en la elaboración de ésta matriz, dejando de lado la condición geográfica y el hecho de que no existe un diseño de matriz marco lógico original para Hambre Cero, es la información disponible para verificar los indicadores propuestos. Por lo general las encuestas de salud, de empleo y condiciones de vida se realizan cada cierto tiempo y no precisamente cada año, además después de cada encuesta pasa un lapso significativo de tiempo para el procesamiento de la información. El siguiente escalón le corresponde a las instituciones, pero como se mencionó anteriormente, estas no tienen sistemas de base de datos ni archivos públicos a la disposición de los ciudadanos, a excepción del Banco Central y el Ministerio de Hacienda.

Contra intuitivamente, no hay evaluaciones del diseño publicadas por parte de los organismos financiadores, en su mayoría son evaluaciones de implementación y que tienen que ver con la transparencia de los procedimientos: rendición de cuentas, criterios de selección de beneficiadas y compra de bienes entregados.

\section{Conclusiones}

La implementación de programas sociales en Nicaragua no está en discusión, más sí el seguimiento que pueda generarse para cada uno de ellos.

Los programas que trabajan bajo el esquema de fondos rotativos suelen tener experiencias exitosas en su implementación por la autosostenibilidad que estos proponen. Sin embargo, el compromiso social con los más vulnerables no finaliza con el hecho de ejecutarlos, también los procesos evaluativos después de la implementación efectivamente son importantes para mejorar la gestión institucional y de recursos empleados en estos programas sociales.

El proceso de construcción de la matriz permitió conocer más a fondo el Programa Productivo Alimentario, pero sobre todo la necesidad de construir un sistema de monitoreo del mismo que actualmen- 
Figura 1: Árbol de Problemas

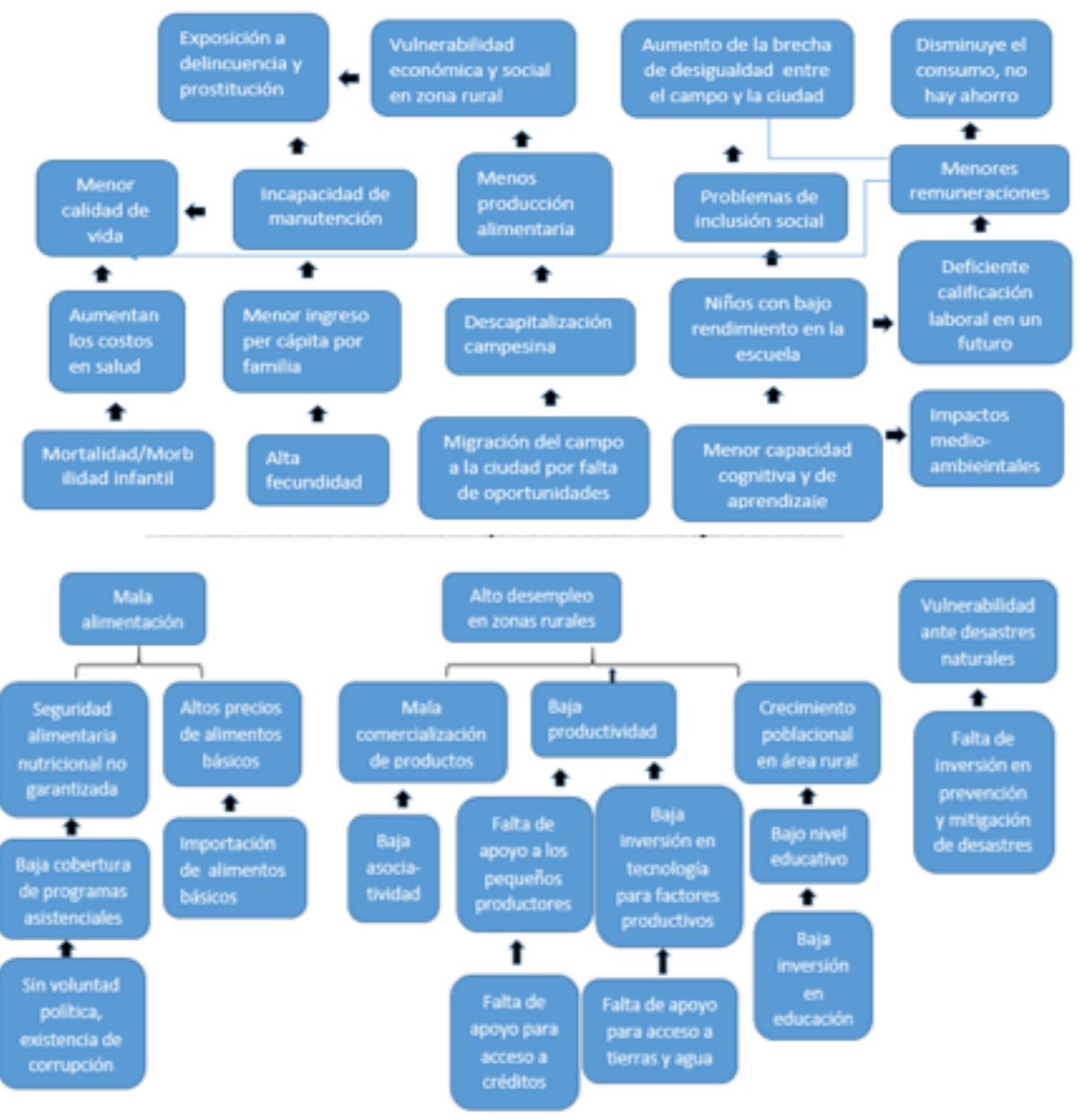

Fuente: Elaboración a partir del documento diseño del Programa Productivo Alimentario de CIPRES y líneas generales de Hambre Cero 
Figura 2: Árbol de Objetivos

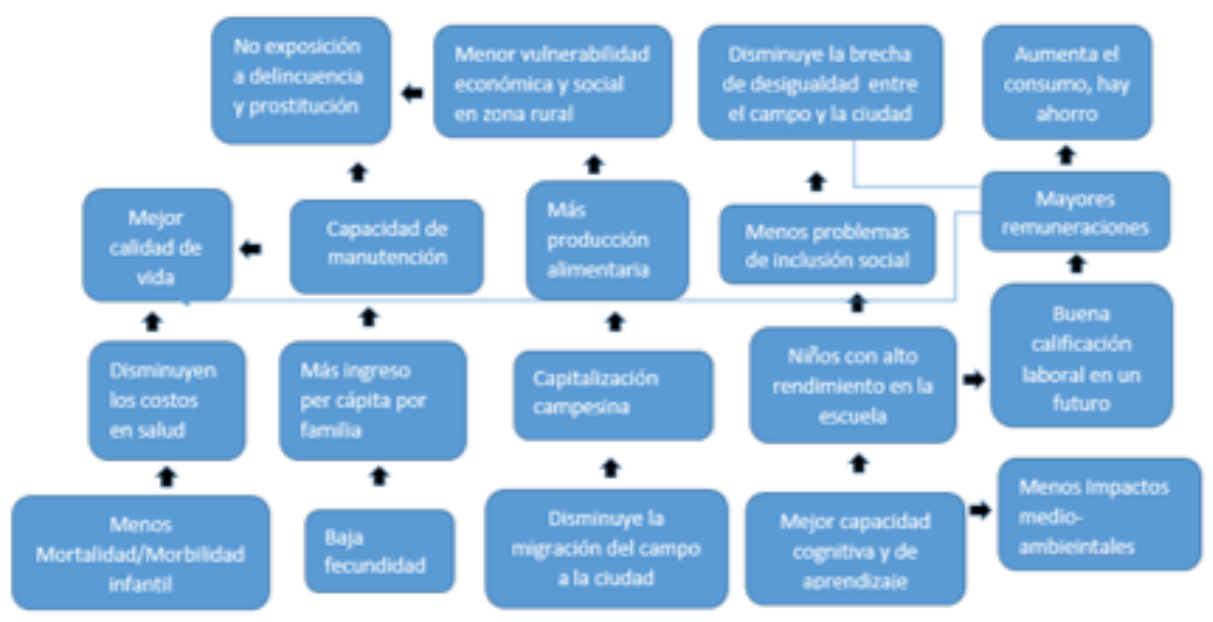

Reducción de población rural en extrema pobreza con hambre y desnutrición
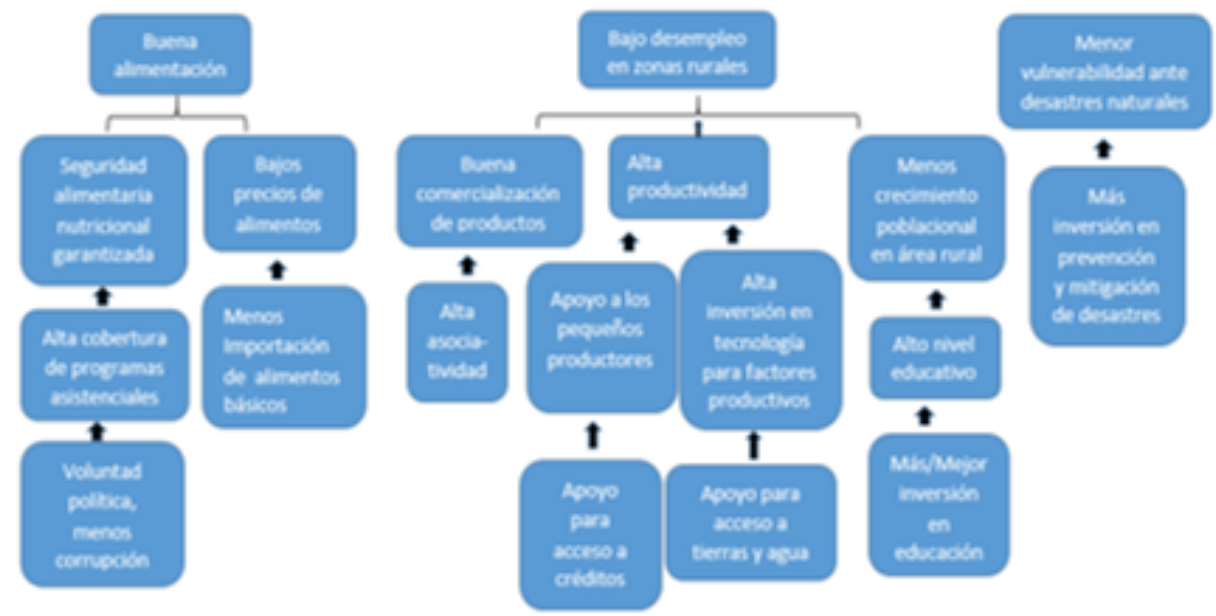

Fuente: Elaboración a partir del documento diseño del Programa Productivo Alimentario de CIPRES y líneas generales de Hambre Cero 
te no existe y que pueda servir de guía para otros programas.

Para elaborar una propuesta de sistema de monitoreo a partir de indicadores basada en marco lógico, fue preciso entender cómo se da el desempeño interinstitucional entre las entidades públicas que han estado involucradas desde el inicio de Hambre Cero, específicamente en cuanto al rol que han ocupado así como su contribución con la información de respaldo que se supone deben tener disponible y proporcionar ante cualquier evaluación integral del programa.

La información es fundamental para cometer los objetivos de construir la propuesta y de medir los indicadores de cumplimiento del programa que ésta plantea; en Nicaragua es un tema sensible pues la información no es de acceso totalmente público como la Ley lo establece. Así pues, es un factor definitivamente determinante para que la propuesta de matriz de marco lógico se materialice, sea efectiva y proyecte los resultados esperados.

Se evidenció una seria deficiencia en los sistemas de información de las instituciones tanto por falta de recursos para construir bases de datos y elaborar informes a partir de estas, así como falta de cultura organizacional de dar seguimiento a las actividades que se van realizando para llevar a cabo los programas.

Diseñar una metodología de este tipo para este y todos los programas sociales en Nicaragua es un gran reto; hay una línea transversal que cruza los procesos que concretan los programas; para revertir una cultura de débil monitoreo y seguimiento se requiere difusión de la importancia y beneficios de usar estas herramientas para el mejoramiento de la gestión y ante la falta de recursos la cooperación internacional juega un papel fundamental en el apoyo de estas iniciativas.

Es posible la puesta en práctica de ésta propuesta, pero ciertamente requiere de serios compromisos asumidos por las instituciones, cambios en la estructura organizacional y profesionalización de los procesos de evaluación.

\section{Recomendaciones}

El proceso de compilación de información respecto a la importancia de dar seguimiento a programas sociales como Hambre Cero, y el estudio del diseño del programa, permiten hacer algunas recomendaciones para la futura implementación del mismo, así como el establecimiento de un sistema de indicadores claros para el monitoreo continuo de éste.
Obviando las deficiencias estructurales de las instituciones y sus funcionarios (Falta de transparencia, casos de corrupción, posible captura política, etc?); a partir de la experiencia de operación del programa, se recomienda elaborar una matriz de marco lógico del programa -acuerdo oficial-, es decir que efectivamente quede establecido como un componente insumo para la evaluación y monitoreo de este.

Una vez establecida la metodología, se requiere de un plan de capacitación de manejo de marco lógico, mismo que sea impartido por expertos contratados en conjunto con el Banco Interamericano de Desarrollo que tiene experiencia trabajando con este enfoque. El Ministerio de Hacienda, que es donde se centralizan los recursos para ser distribuido a demás instituciones del Estado y luego a los demás Ministerios, es donde debería centrarse la implementación de la metodología y que luego demande el uso de ésta en todos los ministerios que ejecutan programas que tienen impacto en la sociedad principalmente.

Definir ésta metodología y forma de operar con un plan operativo anual para realizar las actividades y a partir de esto establecer la forma de monitorear.

Teniendo la evidencia de buenas prácticas de evaluación y monitoreo, se propone establecer, mediante ley, la evaluación de programas sociales, haciéndolo un proceso amigable con los funcionarios, técnicos y profesionales de manera que sean capacitados en Marco Lógico y demás procesos involucrados, institucionalizando el seguimiento de programas en el quehacer del servidor público.

De preferencia, la evaluación debe hacerse por parte de una agencia externa independiente que garantice resultados confiables y evite la politización. Esta agencia debe concursar públicamente, demostrando su capacidad para realizar dicha tarea. Podrían ser paneles de académicos de universidades reconocidas de Nicaragua, expertos independientes, etc.

También se requiere reglamentar la actual ley de acceso a la información pública en cuanto a su cumplimiento. Esto requiere una transformación profunda dentro de las instituciones, debe haber coordinación entre ellas para proporcionar información, así como establecer que ésta se otorgue sin condicionalidades. El que la información sea absolutamente pública exige eficiencia en el cumplimiento de las metas organizacionales y por lo tanto entregar datos de calidad.

Hacer públicas las evaluaciones de monitoreo y seguimiento es fundamental para que la ciudadanía 
conozca y se involucre en los procesos.

Fortalecer la coordinación interinstitucional, consistente con las lecciones aprendidas en Brasil (Fome Zero) y México (Cruzada contra el Hambre) por ejemplo. Se recomienda que se cumpla con la idea de implementación indirecta -a través de ONGs, para reducir los costos y mejorar la efectividad del programa. Además, el programa debe asegurar procesos abiertos y competitivos para la compra, venta, distribución, y transporte de transferencias.

Actualmente la Institución encargada de implementar el Programa Productivo Alimentario es el Ministerio de Economía Familiar, una nueva división del Estado enfocado a fortalecer a la pequeña y mediana empresa tanto rural como urbana. Esta debe publicar informes de gestión periódicos, que permitan mejorar la implementación en el camino y así los resultados presentados sean los deseables.

Crear una línea base del PPA para valorar su impacto real en las beneficiadas y facilitar la realización de diversas evaluaciones, desde su diseño, implementación, hasta una evaluación del desempeño general del programa. En este sentido se propone una sistematización continua de las actividades que se van realizando a fin de documentar los resultados de cada una de ellas.

Realizar flujos de procesos para determinar dónde están los problemas o las dificultades para capturar información requerida en la matriz. Se podría definir una comisión permanente, sea interna o externa, para realizar estos flujos en cada uno de los programas sociales.

Por otro lado, evitar la centralización de la implementación para aumentar su efectividad y disminuir costos, tal como se proponía en un inicio; una iniciativa que integrara a todos los actores locales. En todo el proceso, el PPA fue llevado a cabo con los medios y recursos del Estado, evitando la articulación con programas y proyectos rurales existentes que cuentan con muchos años de experiencia y podrían enriquecer el PPA.

Finalmente, podría ser útil el estudio y revisión de buenas prácticas sobre la utilización del enfoque de marco lógico evidenciado en otros países que han institucionalizado su uso y son testigo de la mejora en la gestión de programas públicos gracias a este.

\section{Referencias}

Banco Central de Nicaragua (2005). Anuario Estadístico 1960-2013, Sector Real. Technical report.

Banco Central de Nicaragua (2013). Informe Anual 2013. Technical report, Managua.

FIDEG (2012). Informe de resultados de la encuesta de hogares. Technical report, Managua.

Food and Agriculture Organization (2012). Revisión y ajuste de Marco Nacional de prioridades para la asistencia técnica de la FAO. Technical report.

GRUN (2012). Plan Nacional de Desarrollo Humano. Technical report.

IEEPP (2011). Hambre Cero- Avances y Desafios. Technical report, Managua.

MAGFOR (2008). Subprograma Productivo Alimentario. Technical report.

Ministerio de Economía Familiar Cooperativa y Asociativa (2012). Programa Hambre Cero. Technical report.

Sabatier, P. (1986). Enfoques de arriba hacia abajo y de abajo hacia arriba en la investigación sobre implementación: un análisis crítico y propuesta de síntesis. Technical report.

Subirats, J. (1995). Los Instrumentos de las políticas, el debate público y el proceso de evaluación. Technical report, México D. F.

UNESCO y SIEMPRO (1999). Gestión integral de programas sociales orientada a resultados: Manual metodológico para la planificación y evaluación de programas sociales. Technical report, Buenos Aires. 